a higher cardiac survival rate than did those who underwent PTCA ( $P=0.008)$. In addition, in the patients with treated diabetes there was a trend towards higher overall survival in the CABG group $(P=0.025)$. These results mirror those published after 7 years of follow-up, when survival among patients with treated diabetes was found to be higher in the CABG group than in the PTCA group.

On the basis of these results, the authors conclude that in patients with multivessel CAD and treated diabetes CABG surgery confers a long-term survival benefit, whereas in patients with multivessel CAD but without known diabetes PTCA and CABG surgery produce equivalent long-term results.

Original article The BARI Investigators (2007) The final 10-year follow-up results from the BARI randomized trial. J Am Coll Cardiol 49: 1600-1606

\section{Biomarker study validates link between trans fat consumption and coronary heart disease risk}

Many dietary assessment studies have demonstrated an association between a diet high in trans fat and an increased risk of coronary heart disease (CHD); however, these studies might not accurately reflect the relationship between trans fat intake and risk of CHD because of measurement errors associated with dietary assessment. Biomarkers have the advantage of being free of such errors. Sun et al., therefore, evaluated the relationship between trans fatty acid content in erythrocytes - a biomarker for trans fat intake-and risk of CHD in 166 incident cases of CHD and 327 matched controls identified from the Nurses' Health Study.

The researchers identified a significant correlation between total trans fatty acid content in erythrocytes and dietary trans fat intake as recorded by food frequency questionnaires. In addition, higher trans fatty acid content in erythrocytes was associated with a significantly increased plasma LDL:HDL ratio-a well-established risk factor for $\mathrm{CHD}$. Following adjustment for age, smoking status, $\mathrm{BMI}$, history of hypertension, and a number of other cardiovascular risk factors, the risk of CHD was 2.7 times higher in women whose total erythrocyte trans acid contents were in the highest quartile than in those whose total erythrocyte trans acid contents were in the lowest quartile.

These biomarker data validate the results obtained from dietary assessment studies, confirming that a high consumption of trans fat is an important risk factor for CHD.

Original article Sun Q et al. (2007) A prospective study of trans fatty acids in erythrocytes and risk of coronary heart disease. Circulation 115: 1858-1865

\section{Rosuvastatin slows intima- media thickening in individuals with subclinical atherosclerosis}

Statins and lifestyle modifications can slow progression or cause regression of atherosclerosis in high-risk populations. The effects of statins on risk of carotid atherosclerosis in low-risk individuals (Framingham risk score $<10 \%)$ are unknown. Crouse and colleagues, therefore, investigated the effects of rosuvastatin on carotid intima-media thickness in a low-risk cohort.

The researchers evaluated progression of atherosclerosis by intention to treat in 984 asymptomatic individuals (mean age 57 years) with moderately elevated LDL cholesterol at low risk of developing cardiovascular disease; people who had ever taken lipid-lowering therapy or had undergone revascularization were excluded. In a 5:2 ratio, participants were randomly assigned $40 \mathrm{mg}$ rosuvastatin or placebo. All participants received therapeutic lifestyle intervention counseling. Intima-media thickness was assessed by carotid ultrasonography before randomization and at 6 , 12,18 and 24 months.

From baseline, rosuvastatin significantly improved patients' lipid profiles compared with placebo. Overall and in individual carotid segments, atherosclerosis progression was significantly slowed in rosuvastatin-treated patients compared with placebo-treated patients (all $P<0.001$ ); however, rosuvastatin treatment was not associated with significant regression, except in the common carotid artery $(P=0.004)$. Progression was seen in all carotid segments in placebo-treated patients, but not in rosuvastatin-treated patients. Few serious adverse events were reported, with similar rates in both treatment groups.

The $40 \mathrm{mg}$ dose selected was chosen to maximize efficacy, but is not a recommended 